\title{
VIEJOS Y NUEVOS MUROS EN EL SISTEMA INTERNACIONAL ${ }^{1}$
}

\author{
Lincoln Bizzozero
}

\begin{abstract}
El trabajo se interroga sobre los cambios que se han producido en el sistema internacional con posterioridad al derrumbe del muro de Berlín. Para ello integra el concepto existencial de "muro" como imposibilidad de llegar a la. lógica del que se encuentra en el entorno inmediato -ya sea local, nacional, estatal o civlizatorio-y analiza los muros derribados, los renovados y los nuevos. El análisis de los muros permite extraer conclusiones acerca de la evolución del sistema internacional en este fin de milenio y de las perspectivas de América Latina frente al mismo.
\end{abstract}

La culminación de la Guerra Fría acarreó en distintos medios académicos y políticos un optimismo ligado a la idea de que la caída del comunismo permitiría imponer la paz y la instauración de regímenes democráticos en el mundo. Esta concepción liberal que se encuentra en el núcleo de una visión de la política exterior de Estados Unidos, se nutre en la base kantiana de que los regímenes democráticos otorgan mayor estabilidad al sistema internacional. De ahí que para esta concepción, la homogeneidad del sistema intemacional en su conjunto, deba asentarse sobre bases representativas y plurales. ${ }^{2}$

Este primer soplo de optimismo una vez que se produjo la caída del muro de Berlín, tuvo como principal exponente a Francis Fukuyama. El "final de la historia" se convirtió en uno de los ejes del debate teórico en los medios académicos, desde distintas aproximaciones disciplinarias. En esencia, su argumentación se centró en la concepción hegeliana de la historia, basada en la idea de que con las revoluciones americana y francesa la humanidad había alcanzado su punto de no retorno en sus

\footnotetext{
${ }^{1}$ Este trabajo fue realizado durante una estadia como profesor invitado para dar unos cursos en la Universidad Laval, en el Institut Québecois de Relations Internationales de la Facultad de Ciencia Politica, durante los meses de octubre y noviembre de 1994: Quiero agradecer a la Universidad Laval, a los profesores del Institut y en particular a su director, Alain Prujiner, el apoyọ recibido.

${ }^{2}$ Michael Doyle, "Kant, Liberal Legacies and Foreign Affairs", Philosophy and Public Affairs, vol. 12, 1983. Del mismo autor puede leerse, "Liberalism and World Politics", American Political Science Review, vol. 80, diciembre 1986.
} 
fundamentos básicos. ${ }^{3} \mathrm{Si}$ bien el artículo de Fukuyama incorpora nuevamente la filosofia en el análisis político, hecho que resulta altamente positivo, las consecuencias de esta visión del sistema internacional pueden ser muy peligrosas y funestas como lo señalara Huntington. ${ }^{4}$

Este impulso "optimista" desde el punto de vista analítico dejó el paso a una etapa "pesimista" marcada por la invasión de Kuwait y la guerra del Golfo Pérsico; la fragmentación y guerra en la ex-Yugoslavia; las derivaciones de la fragmentación de la Unión Soviética; las guerras civiles en Somalia, Ruanda y Yemen. Algunos estudios que surgieron hicieron ver la continuidad y el retorno de la historia: la perspectiva de que las sociedades se enfrentaran a la posibilidad de una nueva Edad Media ${ }^{5}$ desde el punto de vista de sus articulaciones políticas y legitimadoras en el marco de una globalización económica. ${ }^{6}$

El punto culminante de esta etapa se encuentra en el artículo de Samuel Huntington, "The Clash of Civilizations", donde pronostica el conflicto de civilizaciones como paradigma de las relaciones internacionales luego de la culminación de la Guerra Fría. ${ }^{7}$ Esta idea de Huntington se insertó con otras que ya se habían incorporado a los debates europeos sobre el peligro del istamismo en la frontera interna de Europa ${ }^{8}$ y la necesidad de preparación de Occidente a la "nueva cruzada contra los bárbaros". .9 En esta perspectiva, se planteaba un triple frente de batalla:

3 "The End of History", National Interest, verano 1989, y "Réponse à mes contradicteurs", Commentaire, vol. 13, No50, verano 1990. Véase también, la revista Commentaire en sus números 47 , 48,49 y 50, (1989-1990), Paris, donde se hacen diversos comentarios sobre el sentido finalista del articulo de Fukuyama y sobre la perspectiva del futuro del sistema internacional.

${ }^{4}$ Samuel Huntington, "On ne sort pas de l' histoire", Commentaire, vol. 13, No49, Paris, 1990.

${ }^{5}$ Alain Ming. Un nowveau Moyen Age, (Paris: Gallimard, 1993). La idea de un retorno a la Edad Media es percibida desde distintas aproximaciones analiticas. Jean François Revel señala este peligro en "Raison pure et raison pratique", Commentaire, vol. 12, N'48, Paris, enero 1989-1990.

"Véase el debate sobre la aceleración, el retorno, el fin o el cataclismo de la historia en Le Trimestre du Monde, $4^{a}$ trimesire, Paris, 1991. Por otra parte, algunas publicaciones europeas del momento dan cuenta de esa angustia del vacio politico-estratégico y del retomo de la historia. Entre ellas pueden citarse la publicación del Institut Français des Relations Internationales, Ramses 93, (Dunod: Paris, 1992), en especial la introducción y el sugestivo título de la parte segunda "Atonie mondiale et dynamismes régionaux"; y François Joyaux, "Actualité stratégique", Documents Payot, Toulouse, 1992.

${ }^{7} \mathrm{El}$ artículo apareció en Foreign Affairs, en el vol. 72, $\mathrm{N}$ 3 , verano de 1993. Tuvo respuestas de varios autores en el número siguiente de la revista (vol. $\left.72, \mathrm{~N}^{\circ} 4\right)$. Por otra parte, la revista Commentaire dedicó el $\mathrm{N}^{\circ} 66$, verano de 1994 , a comentar el artículo por varios especialistas.

${ }^{8} \mathrm{El}$ "dilema" civilizatorio como peligro ya habia sido señalado en la década del ochenta por algunos analistas europeos, entre los cuales se encuentra Régis Debray. La novedad de Samuel Huntington, es de incorporarlo como paradigma explicativo de las relaciones internacionales.

9 Jean-Christophe Rufin, L'empire et les nouveawx barbares, (Jean-Claude Lattès: Paris, 1991). De acuerdo a esta concepción, la caída del imperio soviético deja en pie solamente a los nuevos bárbaros representados en un multiforme Tercer Mundo. Esos "bárbaros" obedecen a otro cuerpo de creencias y códigos del que tiene Occidente, por lo que pueden socavar sus bases como ocurriera con el imperio 
el que surgía de la vígilancia internacional frente: a cualquier violación de la legalidad intemacional, como fue el caso de la invasión de Kuwait por parte de Irak; el que prövenía de läs relaciones bilaterales y multilaterales, donde se consideraba fundamental el rol de las potencias en la observancia del respeto de los derechos del hombre, como lo planteara Francia en la cumbre franco-africana de La Baule en 1990; y; finalmente; el cuidado del frente interno, que implicaba una supervisión para que instituciones o actores nacionales no se prestaran a intereses espúreos.

Por último, recientemente se inició una tercera etapa de procesamiento de los distintos problemas. Esta etapa de revisionismo convergió con una modificación de la política exterior de Bill Clinton y un estancamiento estratégico sobre las principales opciones a encarar en los distintos conflictos y en el marco de las relaciones entre el sistema internacional y los Estados. 10

Entre estas etapas de "optimismo", "pesimismo", y "revisionismo inmovilista" en la post-Guerra Fria, se sucedieron un conjunto de acontecimientos y de debates con diferentes niveles de importancia, que han incorporado su marca en el diseño del sistema intemacional. Resulta claro que muchos de esos acontecimientos se precipitaron como consecuencia de la caida del muro ideológico. Esta serie de hechos ligados en una suerte de efecto dominó, tienen de por sí validez propia y resultan pertinentes en cuanto a sus contenidos y consecuencias. Sin embargo, de esta sucesión de acontecimientos en efecto dominó en distintos campos y dominios no pueden extraerse reglas generales de una definición del contenido del sistema internacional futuro, ni de sus consecuencias de base en el funcionamiento vinculadas al "optimismo" o "pesimismo". 11

romano. La otra posibilidad histórica es que terminen sojuzgados. La guerra del Golfo Pérsico corresponderia a la primera fase de esta nueva cruzada. Desde una perspectiva económica, la inmigración de trabajadores y la reubicación productiva trajo consigo la angustia al mundo occidental frente a la invasión de los bárbaros. Ver al respecto, Julien Freund, La fin de la Renaissance, (Paris: 1980).

${ }^{10}$ Sobre la revisión de Bill Clinton de la poitica exterior americana de la post-Guerra Fría, véase Roberto Russell, "La politica internacional de Bill Clinton, el sistema internacional y América Latina: notas para un debate", en: Lincoln Bizzozero, Klaus Bodemer, Marcel Vaillant (coord.), Nuevos regionalismos: cooperaciọn o conflicto?", (Universidad de la República, Nueva Saciedad: Venezuela, 1994). El estancamiento estratégico es una dé las conclusiones del informe aniual del IISS, Strategic Survey, (Brassey's: Londres, 1994). Véase también Nicole Gnesoito, "Deux" fois deux mondes", en: Thierty de Montbrial, Pierre Jacquet, Ramses 95. Sythèse annuelle de l'actualité mondiale, (Paris: 1994).

${ }^{11}$ Los conceptos "optimismo" $y$ "pesimismo" han hecho su camino entre los analistas sociales. Basta recordar entre otros algunos trabajos de Hirschman para decantar toda idea cuestionadora a los anälisis con una evaluación subjetiva sobre posibles escenarios futuros. Es por otra parte significativo el hecho de que el público se ubique en ese eje para interrogarse sobre los acontecimientos internacionaies. En la conferencia del Profesor Franz Nuscheler "El conflicto Norte-Sur: ¿fin o 
Existen, eso sí, distintas manifestaciones, que están definiendo las formas y modalidades del sistema internacional, y es en esa ingeniería que tienen que reposar las explicaciones pertinentes sobre el funcionamiento, los paradigmas y los conceptos-base.

A efectos de no caer en una enumeración de acontecimientos y para guardar un eje orientador, vinculado con los debates sobre "optimismo" y "pesimismo" en el sistema internacional, se ha buscado incorporar el concepto existencial de "muro". Esta opción analítica, tiene un contenido social universal: el muro define la imposibilidad de llegar al Otro; al problema de enfrente; a la lógica y a los mecanismos de elaboración del que se encuentra del otro lado de mi entorno inmediato (local, nacional, estatal o civilizatorio en un sentido general).

Esta reflexión analítica se asienta en varias interrogantes que han surgido a lo largo de estos últimos años, con posterioridad a la caída del muro de Berlín. Para ser más precisos, los interrogantes sobre el alcance de los cambios post-muro terminaron de plasmarse en una exposición con posterioridad a las elecciones en Africa del Sur. ${ }^{12}$ En particular, las preguntas pueden resumirse en los siguientes puntos: ¿cuáles son los muros que se han derrumbado en estos últimos años?; ¿cuáles son los nuevos muros que han surgido?; qué consecuencias y perspectivas puede aparejar para el sistema internacional?; y, ¿cómo ubicamos en ese contexto a América Latina?

En el desarrollo de estas interrogantes nos remitiremos en primer lugar a señalar los distintos muros que han sido derribados y que dejan un margen de "optimismo" en las relaciones internacionales. Los muros que han caído en estos últimos años son: el "muro de Berlín", el apartheid, el de las "relaciones israelo-palestinas", el "nuclear", el "ideológico-político", el del reconocimiento entre los Estados del Vaticano e Israel y el de la integración entre países de distinto nivel de desarrollo. En segundo orden nos referiremos a los nuevos muros que se han desarrollado en el sistema intemacional en estos últimos años, aun cuando todavía no están definidos sus contomos y la profundidad de los mismós. Estos nuevos muros son: el "civilizatorio", el "nacional", el "Este-Oeste", el "Norte-Sur" y finalmente el que procede de la "amenaza interna".

agravamiento?", que se llevó a cabo en Montevideo en el Instituto Góethe, una pregunta pertinente se centró en la evolución del "optimismo" frente a varios sucesos internacionales.

${ }^{12} \mathrm{La}$ exposición fue realizada por Aureliano Rodriguez Larreta en el Centro Latinoamericano de Economía Humana. Con posterioridad a la misma, comentamos con Romeo Pérez Antón la idea de la división histórica entre los "viejos" y los "nuevos" muros, en el sistema internacional. 
La evaluación de estos distintos acontecimientos en relación a las relaciones internacionales de la humanidad tiene consecuencias indudables en este final del milenio. Por otra parte, la inserción de América Latina en el sistema intẹnacional y su proyección en términos de evaluaciones rendidoras y competentes, no está ausente de estos debates y de la persistencia de muros en la comunidad internacional.

\section{I.- Derrumbes y renovaciones.}

Como se señaló recientemente, los muros que fueron derribados últimamente son: el "muro de Berlín", el apartheid, el de las "relaciones israelitas-palestinas", el "nuclear", el "ideológico-político", el del reconocimiento entre los Estados del Vaticano e Israel, el de la integración entre países de distinto nivel de desarrollo. La caída de algunos de esos muros permitió una reconstrucción nacional o en el seno de un Estado, como fueron los casos del "muro de Berlin", el "apartheid" y el muro "ideológico-político". En otros casos, el derrumbe del muro posibilita abrigar esperanzas serias vinculadas con la seguridad y la paz en una región (muro israelita-palestino) o en el mundo (muro nuclear). Finalmente, la caída de algunos muros permite aproximaciones de dos creencias (acuerdo Vaticano-Estado de Israel) o entre países de distinto nivel de desarrollo (Tratado de Libre Comercio de América del Norte).

\section{Las reconstrucciones.}

En los muros que permitieron las reconstrucciones encontramos el núcleo duro que posibilitó la reformulación del sistema internacional. En particular, la caida del muro de Berlín no deja de ser un símbolo, tanto por su significado como por la vitalidad y rapidez con que se efectuó la reconstrucción del espacio alemán. El proceso de reunificación alemán duró en definitiva desde el 9 de noviembre de 1989 hasta el 2 de diciembre de 1990, día de la elección del primer Parlamento panalemán.

El muro separó durante cuarenta y cinco años dos Estados con una misma pertenencia nacional y por otra parte dividió a Alemania del resto de Europa. Esta doble separación se acompañó de varias tutelas sobre Alemania: las tropas de ambos bloques se encontraban estacionadas de los dos lados del muro; la frontera "caliente" de la Guerra Fría estuvo continuamente bajo vigilancia; en caso de guerra convencional o nuclear, Alemania sería la primera afectada; y era en el territorio alemán donde 
se percibían los efectos inmediatos de la competencia entre regímenes contrapuestos.

La agenda de temas externos vinculados a la reunificación fue dificil y compleja. Entre los diversos temas a resolver se ubicaron el vinculado al estatuto político y militar de Alemania, ligado a la pertenencia nacional a Europa Occidental o conformación de una entidad diferenciada, la presencia de tropas extranjeras, los derechos de las cuatro potencias victoriosas de la Segunda Guerra Mundial, un arreglo con los vecinos en términos fronterizos y de seguridad. Sin embargo, este conjunto de temas que tenían relación con el muro, y que hubieran retardado el proceso de reunificación, debieron ser resueltos rápida y prácticamente, porque el empuje reunificador del Parlamento de la República Democrática Alemana (RDA), formado después de las primeras elecciones libres realizadas el 18 de marzo de 1990, fue incontenible. En efecto, así como la expresión popular empujó a la apertura anticipada del muro en manifestaciones populares de envergadura, ${ }^{13}$ el resultado de las elecciones en la RDA derivó en una petición de fusión inmediata, que llevó al Canciller Kohl a la decisión de fijar las elecciones conjuntas en diciembre del mismo año.

La decisión del Canciller Kohl aceleró las instancias necesarias previas a la elección conjunta, tanto en términos de la reunificación como de las relaciones con los Estados vecinos y las potencias victoriosas. El 18 de mayo se firmó el Tratado de Unión monetaria, económica y social, mediante el cual se consagró la extensión de la economía social de mercado. El 31 de agosto se firmó el Tratado de Unificación, sobre la base del artículo 23 de la Ley Fundamental del 8 de mayo de 1949, el cual fue ratificado a las dos semanas por ambos Parlamentos. ${ }^{14}$

La misma Ley Fundamental señalaba, en su artículo 23, que la Ley se aplica a todo el territorio de los lander enumerados recientemente y

\footnotetext{
${ }^{13}$ Las manifestaciones inmediatamente después de la abolición de la frontera pasaron de la consigna "nosotros somos el pueblo" a "nosotros somos un pueblo", proclamando de hecho la unidad. Por otra parte, el número de alemanes que pasó al lado occidental se acrecentó enormemente (130.000 en noviembre), planteando el problema de la reconstitución en términos inmediatos. Véase Jean Klein-Walter Schûtze, "La quẹstion allemande", en: Thierry de Montbrial (dir), Rapport Annuel Mondial sur le Système Economique et les Stratégies 91, (IFRI, Dundod: Paris, 1990.

${ }^{14}$ La rapidez de la reunificación alemana fue posibilitada por las disposiciones de la Ley Fundamental del 8 de mayo de 1949, que señalaba en su preámbulo "...el pueblo alemản de los Estados (lander) de Baden-Wurtemberg, Baja Sajonia, Baviera, Brema, Hamburgo, Hesse, Renania-Palatinado, Renania-Westfalia, Sarre y Schleswig-Holstein, con la finalidad de dar un orden nuevo a la vida política, durante este periodo transitorio ha adoptado, en virtud de su poder constitucional, la presente Ley fundamental de la República Federal de Alemania. Ha actuado igualmente en el nombre de los Alemanes, a los que se les impidió actuar en esta tarea. El pueblo alemán en su conjunto, disponiendo libremente, queda invitado a concluir la libertad y la unidad de Alemania".
} 
concluye que en las otras partes de Alemania la ley estará vigente una vez que se produzca la accesión. El 12 de septiembre, los participantes de la Conferencia " $2+4$ " firman el acuerdo definitivo sobre Alemania. El 3 de octubre es proclamada en Berlín la soberanía, luego de que las cuatro potencias terminaran con sus responsabilidades derivadas de los acuerdos de Potsdam. Finalmente, este proceso culmina con las elecciones en el conjunto de Alemania reunificada.

Aparte de esta reunificación, los acuerdos de buena vecindad con la Unión Soviética, los acuerdos fronterizos y de cooperación con los países de Europa Central y el reconocimiento de la pertenencia de Alemania a la Alianza Atlántica, por la cual se reafirmó la asociación en la dirección, terminaron de enterrar las secuelas del muro en términos de la historia reencontrada.

Es indudable, que la reunificación abrió inmediatamente otra àgenda de temas dificiles, tanto al interior de Alemania como en sus relaciones con los países vecinos y en sus compromisos en el contexto de Europa Occidental. En términos del proceso alemán en sí mismo, la reunificación trajo consigo todas las consecuencias inherentes a la absorción de una sociedad alemana con otro nivel de desarrollo económico y en particular con otra cultura societal de filosofia económica. 15

En lo que concierne a este trabajo, no interesan las consecuencias de la reunificación ni tampoco realizar un análisis específico del impacto en términos del sistema internacional, salvo que del mismo se derivaran situaciones que llevaran a lógicas de creación de nuevos muros. ${ }^{16}$ Este efecto no se produjo, debido al sistema político que fue capaz de canalizar en los difíciles momentos post-transición las reivindicaciones de los distintos grupos de los nuevos lander alemanes y de realizar redistribuciones panalemanas sin cuestionamientos legitimadores desde los lander occidentales. ${ }^{17}$

\footnotetext{
${ }^{15} \mathrm{La}$ lógica de la continuidad de un muro intraalemán es señalada por algunos analistas, entre los cuales, Klaus Schubert, "Ce mur dans les têtes", Géopolitigue, No44, invierno 1993-94. Por otra parte, esta dificultad de asimilación de dos culturas con lógicas económicas que se encuentran en las antipodas, ya había sido señala da por ...filosofĩa económica... En efecto, el libre mercado, como cualquier otro funcionamiento societal de la economia, no se crea por decreto. Los problemas del inicio de la reunificaciön pueden verse en Françoise Nicolas, "L'Allemagne unifiée, des débuts difficiles", en:.Thierry de Montbrial (dir.), Ramses 92. Système économique et stratégies. Le monde et son évolution", (IFRI, Dunod: Paris, 1991).

${ }^{16}$ Este podria haber sido el caso si la reunificación hubiera derivado en una lógica nacionalista-xenófoba. Hasta el momento sin embargo, los partidos aseguran la vigencia del pluralismo y el reconocimiento de las diferencias. Por otra parte, el ciudadano alemán ha seguido apostando a esa tradición, como lo atestiguan las recientes elecciones.

${ }^{17}$ Véase al respecto Klaus Bodemer, "Alemania", en: Helio Jaguaribe (coord.), La sociedad, el Estado y los partidos politicos en la actualidad brasileña, (F.C.E.: México, 1992).
} 
La caída del muro del apartheid en Africa del Sur también posibilitó un reencuentro cívico de una mayoría marginada de sus derechos y obligaciones en un contexto estatal. La similitud histórica con el muro alemán no proviene solamente del contexto sistémico internacional en que se desarrollaron ambos muros, sino también de su resolución refrendada popularmente. El pueblo alemán festejó mayoritariamente la caída del muro de Berlín, como así también las mayorías negras marginadas de Africa del Sur celebraron la realización de las elecciones y el triunfo de Mandela.

El derrumbe del muro del apartheid tuvo distintas etapas, pero también en este caso la rapidez y vitalidad del proceso no posibilitaron los intentos de frenarlo, tanto por parte de la extrema derecha blanca como de los zulúes. ${ }^{18}$ En términos del proceso desencadenante, el reencuentro de la mayoría con sus derechos y deberes cívicos fue posibilitado primeramente por las reformas encauzadas por el gobiemo de Frederik De Klerk, desde febrero de 1990. Con posterioridad, fue la propia minoría blanca que otorgó en un plebiscito en marzo de 1992 el respaldo al gobiemo para continuar en la vía de las reformas, con el objetivo de reformar la Constitución y en definitiva el funcionamiento del país.

La aceleración del cambio se produjo a partir de 1993: diálogo entre distintos partidos en el marco de la CODESA (Convention on a Democratic South Africa); aprobación de una Constitución transitoria por parte de la CODESA en noviembre; formación de un Consejo ejecutivo provisorio que aseguraría la transición compuesto por veinte partidos políticos en diciembre; elecciones generales en abril de $1994 .{ }^{19}$

También en Africa del Sur la caída del muro provocó la incorporación de nuevos temas en la agenda gubernamental. Entre ellos, la posibilidad de una segregación de la minoría blanca y de los zulúes; la necesidad de realizar cambios para atender los enormes desequilibrios económicos, sin provocar desequilibrios ni desestabilización en las directivas de la política económica. 20

\footnotetext{
${ }^{18}$ Los partidos Conservador de Ferdi Hartzenberg y el Movimiento de Resistencia Afrikaner AWB de Eugène Terreblanche, crearon el Frente del Pueblo Afrikaner (Afrikaner Volksfront) en mayo de 1993, para contrarrestar la perspectiva del gobierno por la mayoría. Por otra parte, los zulúes tenian su propio partido, el Inkatha Freedom Party del jefe Mangosuthu Buthelezi.

${ }^{19}$ Para una historia del desmantelamiento del apartheid, véase J. Rigault, Le demantèlement de l'apartheid", (L' Harmattan: Paris, 1992). Los detalles de la negociación, sus avances e incertidumbres, pueden seguirse en los articulos de Pierre Haski en L'Etat du Monde. Annuaire économique et géopolitique mondial, (La Découverte-Boréal: Paris, 1992, 1993 y 1994).

${ }^{20}$ Las dificultades del dia después del apartheid han sido analizadas en distintos trabajos y articulos. Como mención puede leerse el articulo de Vincent Maphai, "Prospects for a democratic South Africa", International Affairs, vol. 69, N22, abril de 1993.
} 
nización. Sin embargo, las elecciones generales realizadas en 1992, luego de un acuerdo de paz firmado en mayo de 1991, fueron cuestionadas por el grupo guerrillero UNITA, impidiendo en este caso el encuentro de los distintos beligerantes en el marco estatal. ${ }^{25}$

En Camboya, la firma de los acuerdos de París en octubre de 1991, colocaron al país bajo la tutela de las Naciones Unidas hasta la realización de elecciones libres. Estos acuerdos aceleraron los pasos internos para la reconstrucción nacional. Entre la firma de los acuerdos de París y la realización de elecciones en mayo de 1993, se sucedieron varios acontecimientos de importancia en el contexto "derrumbe muro" y reconstrucción nacional: repatriación de refugiados; creación por parte de las Naciones Unidas de una Autoridad Provisoria, desarme de las tropas que respondian a las distintas fracciones, sanciones a los khmers rojos por parte del Consejo de Seguridad de las Naciones Unidas. El elevado porcentage de participación en las elecciones (alrededor de $90 \%$ ), a pesar del sabotage de los khmers rojos, demostró el entierro de la etapa anterior por parte de los camboyanos. Las actuales dificultades de empantanamiento, responden a la situación institucional y política vinculada con los equilibrios personales de poder y con el estado de salud de Sihanouk.

\section{Los procesos de pacificación y la seguridad.}

El acuerdo de Oslo entre Israel y la Organización para la Liberación de Palestina (OLP), firmado por Itzhak Rabin y.Y Yasser Arafat en septiembre de 1993, se inscribe en un marco general de negociaciones multilaterales entre árabes e israelitas. También en esta situación, el inicio de las negociaciones en Madrid en octubre de 1991, tuvo como lógica implícita terminar con un status quio: en este caso, el heredado en la región luego de la caída del imperio otomano.

Los problemas específicos fueron abordados desde el inicio de las negociaciones. En Madrid, fueron definidos los cuatro grupos de trabajo que correspondían a los expedientes conflictivos. Los temas encarados fueron: la cooperación económica, el control de armamentos y la seguridad regional, el ambiente y los recursos hidráulicos. A estos cuatro expedientes, se agregó en la reunión de Moscú el correspondiente a los refugiados. Si bien el avance en casi todos los grupos tiene un techo vinculado con la ausencia de algunos de los Estados de la región, entre

25 Véase la crónica de los acontecimientos y negociaciones tanto en Angola como Mozambique en Hugo Sada, "Afrique subsaharienne", en: Pascal Boniface (dir.), L'année stratégique 1993. Les équilibres militaires, (Dunod: París, 1993). 
nización. Sin embargo, las elecciones generales realizadas en 1992, luego de un acuerdo de paz firmado en mayo de 1991, fueron cuestionadas por el grupo guerrillero UNITA, impidiendo en este caso el encuentro de los distintos beligerantes en el marco estatal. ${ }^{25}$

En Camboya, la firma de los acuerdos de París en octubre de 1991, colocaron al país bajo la tutela de las Naciones Unidas hasta la realización de elecciones libres. Estos acuerdos aceleraron los pasos internos para la reconstrucción nacional. Entre la firma de los acuerdos de París y la realización de elecciones en mayo de 1993, se sucedieron varios acontecimientos de importancia en el contexto "derrumbe muro" y reconstrucción nacional: repatriación de refugiados; creación por parte de las Naciones Unidas de una Autoridad Provisoria; desarme de las tropas que respondian a las distintas fracciones; sanciones a los khmers rojos por parte del Consejo de Seguridad de las Naciones Unidas. El elevado porcentage de participación en las elecciones (alrededor de $90 \%$ ), a pesar del sabotage de los khmers rojos, demostró el entierro de la etapa anterior por parte de los camboyanos. Las actuales dificultades de empantanamiento, responden a la situación institucional y política vinculada con los equilibrios personales de poder y con el estado de salud de Sihanouk.

\section{Los procesos de pacificación y la seguridad.}

El acuerdo de Oslo entre Israel y la Organización para la Liberación de Palestina (OLP), firmado por Itzhak Rabin y.Y Yasser Arafat en septiembre de 1993, se inscribe en un marco general de negociaciones multilaterales entre árabes e israelitas. También en esta situación, el inicio de las negociaciones en Madrid en octubre de 1991, tuvo como lógica implícita terminar con un status quio: en este caso, el heredado en la región luego de la caída del imperio otomano.

Los problemas específicos fueron abordados desde el inicio de las negociaciones. En Madrid, fueron definidos los cuatro grupos de trabajo que correspondían a los expedientes conflictivos. Los temas encarados fueron: la cooperación económica, el control de armamentos y la seguridad regional, el ambiente y los recursos hidráulicos. A estos cuatro expedientes, se agregó en la reunión de Moscú el correspondiente a los refugiados. Si bien el avance en casi todos los grupos tiene un techo vinculado con la ausencia de algunos de los Estados de la región, entre

25 Véase la crónica de los acontecimientos y negociaciones tanto en Angola como Mozambique en Hugo Sada, "Afrique subsaharienne", en: Pascal Boniface (dir.), L'année stratégique 1993. Les équilibres militaires, (Dunod: París, 1993). 
los que se encuentran Siria y Líbano, la definición del acuerdo permite encarar con alguna expectativa la caída del muro mayor árabe-israelita en términos de la seguridad regional.

De esta manera, el Acuerdo de Oslo entre Israel y la OLP como representante del pueblo palestino, permite definir los dos planos en que ese Acuerdo tiene repercusiones: el vinculado con las negociaciones árabe-israelitas para una seguridad regional y el relacionado con el tema palestino. Ambos planos tienen sus respectivas dinámicas, aunque se encuentren interconectados y se condicionen mutuamente.

En el plano de las negociaciones regionales, falta un trecho importante a tecorrer, aun cuando los avances realizados han sido importantes y permiten encarar con una expectativa cierta la caída del muro árabeisraelita en términos de la seguridad regional. Es así, que con posterioridad al Acuerdo de Oslo, Israel inició negociaciones con Jordania, que čulminaron en un acuerdo específico. Ahora se presenta por delante el problema más dificil en términos de la real politik: el de las relaciones entre Siria e Israel. ${ }^{26}$ Es en este tramo del muro que las perspectivas de avanzar en materia de seguridad regional encuentran dificultades, aunque las tendencias alimentan la continuidad del proceso.

Sin embargo, para llegar a derribar este núcleo duro deben tenerse presente algunos puntos que la perspectiva del "optimismo" hace olvidar. Entre los mismos deben señalarse los siguientes: los equilibrios regionales y la defensa del interés nacional obedecen a una lógica independiente del que tenía el conflicto Este-Oeste; a estos equilibrios basados en regulaciones nacionales deben agregarse problemas fronterizos y nacionales no resueltos; los cortes y divisiones religiosos no corresponden a los cortes nacionales; finalmente el resurgimiento del islam y del fundamentalismo tiene una lógica propia que puede bloquear los otros niveles.

Este primer plano no soslaya los avances realizados en el segundo plano, que de por sí son relevantes. Entre esos avances vinculados con el primer plano, pero que han derivado en consecuencias especificas post-muro, se encuentran: el reconocimiento de la OLP por parte del Estado de Israel, tanto en términos de su legitimidad palestina como de su capacidad para gestionar la transición de los territorios autónomos y el reencuentro entre palestinos en esos territorios. ${ }^{27}$

\footnotetext{
${ }^{26}$ Acá debemos ubicar entre las dificultades las "objetivas". y las "subjetivas". Entre las primeras hay que señalar especificamente los temas del Libano y del Golán, que no pueden tener la misma solución que la concerniente a los territorios de Gaza y Cisjordania. Entre las dificultades "subjetivas" se ubica la percepción del otro y el tema de la confianza, por lo que el "muro" en términos de una paz regional sigue vigente.

${ }^{27}$ Hay que señalar, sin embargo, que el grupo de trabajo sobre los refugiados no avanzó mucho, por lo que quedan en negociación la caracterización del refugiado, su vinculación con los distintos
} 
Todavía resulta prematuro poder realizar una evaluación de la gestión palestina y del debate al interior de la OLP, cuyo desenlace parece incierto. Entre los diversos aspectos a orientar y regular se encuentran: la normalización de la vida política; la formación de instituciones democráticas que permitan la expresión de los diversos componentes del pensamiento palestino; el aprendizaje de una gestión administrativa independiente que permita forjar las bases del futuro Estado. Por otra parte, los cuestionamientos a la OLP desde las propias tiendas árabes y palestinas continúan, tanto sobre su representatividad como sobre el contenido o los diversos puntos de la negociación. Estas dificultades de gestión internas de la OLP son percibidas como una traba al asentamiento de bases más seguras en la región por parte de la opinión pública israelí. ${ }^{28}$

Los avances en este plano no permiten predecir avances sustantivos en materia de canales de comunicación a través del muro civilizatorio. Esta diferenciación de muros, de planos y de aproximaciones a diferentes problemáticas, permite explicar la continuidad de negociaciones a pesar de los atentados terroristas. Estos distintos muros, explican también la perspectiva de "sociedades separadas" propuesta por el Primer Ministro de Israel, Yitzhak Rabin, en represalia a los atentados del grupo Hamas. ${ }^{29}$ En definitiva, esta propuesta puede obrar como disparador al interior de la sociedad palestina, ya que hay alrededor de 80.000 palestinos de los territorios autónomos trabajando en Israel, en una población que no llega a los dos millones, pero también puede promover la interrupción del proceso subregional. Al respecto importa también tener en cuenta la evolución de las perspectivas nacionales. En Israel, con posterioridad al atentado de Tel Aviv, el $71 \%$ de la opinión pública se decía partidaria de una intervención directa en Gaza, contrariando de esa manera el mismo acuerdo firmado con los palestinos. ${ }^{30}$

En síntesis, el dernumbe del muro israelí-palestino es de por sí un paso importante en la opción por modificar el status quo de la región, aunque queden varios expedientes a resolvery la necesidad de incorporar

\footnotetext{
hechos históricos y el número de los mismos. Sin embargo, el punto vinculado con el principio de la reunificación de las familias fue acordado. Ello explica el retomo de miles de personas a Cisjordania y Gaza entre 1992 y 1994.

${ }^{28}$ Solamente una minoria de la opinión pública cree que la OLP es capaz de controlar los extremistas, aun cuando un $60 \%$ considera a la OLP como el interlocutor adecuado. Véase Bassma Kodmani-Darwish, "Moyen-Orient: la marche irréversible vers la paix?", en: Thierry de Montbrial, Pierre Jacquet (dir), op.cit.

${ }^{29}$ Inmediatamente después del atentado, el gobiemo decidió traer 15.000 obreros extranjeros y no prolongar el permiso de residencia por un año de varios miles empleados en Israel, según los cables de AFP del viernes 21 de octubre de 1994.

${ }^{30}$ Véase el editorial de François Brousseau, "Séparatism proche-oriental", Le Devoir, del 21 de octubre de 1994, Montreal, Canadá.
} 
otros actores. En términos microregionales, la caída del muro tuvo varios efectos: la autonomía de los territorios ocupados, el reconocimiento de la OLP, el reencuentro de los palestinos en los territorios autónomos y la resolución política del tema de la intifada. En prospectiva, los avances en la pacificación regional tienen dos techos: el derivado de la real-politik, que en la región es un juego de varios actores, y el civilizatorio.

El derrumbe del muro nuclear también se relaciona con las modificaciones del ambiente internacional post-muro de Berlín. En este dominio, el conjunto de acuerdos de desarme impresionan tanto por su amplitud como por la rapidez con que se procesaron las distintas iniciativas. La ratificación del acuerdo STARTI por parte de los Estados Unidos, Rusia, Kazakhstán, Bielorusia y Ucrania, luego de algunas incertidumbres iniciales, culminó veinticinco años de negociación sobre el tema. ${ }^{31}$

El derrumbe de este muro, que también se aceleró en los últimos años, tiene varias facetas. En Europa permitió una distensión y el reencuentro entre el sistema político, los decisores y la sociedad civil, después de ese quiebre en materia de consenso sobre la seguridad que había significado la introducción de los euromisiles. Es indudable, que las consecuencias de este derrumbe introducen otros problemas en materia de seguridad, que todavía no han sido resueltos. Lo sustantivo, sin embargo, en el Viejo Continente, es que el debate actual no pasa por la separación sociedad civil-élite de decisores y sobre la posible utilización del ama nuclear a nivel regional (armamento táctico de medio alcance), sino por la definición de estructuras adecuadas en las instituciones vigentes, con objetivos, roles y funciones definidos en cuanto a la seguridad regional.

En el conjunto del sistema internacional, los diferentes acuerdos de desarme permitieron descartar la hecatombe nuclear como amenaza inmediata. También en este nivel, el derrumbe del muro nuclear como amenaza ha tenido efectos específicos. Ello se percibe tanto en el ciudadano mundial, como en la definición de los temas de seguridad por parte de los Estados. En lo que se refiere al ciudadano mundial, la desaparición de la amenaza nuclear le posibilita tener una visión alternativa a un final de la historia. Por otra parte, el ciudadano nacional ( $y$ de los países del Tercer Mundo), se ha visto obligado a desligar el tema del desarrollo de la confrontación de dos modelos alternativos en términos estratégicos. Ello quiere decir, lisa y llanamente, que los propios códigos

\footnotetext{
${ }^{31}$ Las incertidumbres iniciales fueron resueltas favorablemente en las negociaciones entre Estados Unidos, Rusia, Bielorusia y Kashakstán. Los problemas se mantuvieron con Ucrania, hasta que firmó el tratado el 18 de noviembre de 1993, con trece condiciones.
} 
internacionales obligan a que las sociedades nacionales (y también del Tercer Mundo), replanteen sus objetivos, modelos, alternativas y perspectivas en materia de desarrollo. ${ }^{32}$ En cuanto a los temas de seguridad que se encuentran presentes en la actualidad entre los Estados, los mismos tienen más en cuenta el plano regional que el internacional.

Finalmente, los acuerdos ratifican la supremacía de los Estados Unidos y Rusia en el dominio nuclear, aun cuando otros Estados dispongan de la tecnología necesaria. Las reducciones en curso entre ambos, cuando culminen, definirán una proporción diferencial de ocho a uno en relación a las otras potencias nucleares. Por lo tanto, el derrumbe del muro nuclear no traerá aparejado la caducidad del arma nuclear como referente de vigilancia estratégico en el sistema internacional.

La rapidez y amplitud de los avances en el desarme tienen otra derivación vinculada con el control del factor nuclear. La Conferencia de 1995 de las partes del Tratado de No Proliferación de armamento nuclear, deberá definir su continuidad o revisión. En principio, las potencias nucleares están de acuerdo en darle continuidad al Tratado, pero algunos países cuestionan la asimetría del mismo y quieren modificar algunos supuestos. Por otra parte, algunos vacios en materia del control nuclear tanto a nivel internacional como nacional han planteado el tema de la seguridad en términos regionales.

En una perspectiva general, el objetivo de llegar al umbral nuclear ha sido descartado por varios Estados, entre los que se cuentan Argentina y Brasil. Por otra parte, Africa del Sur, dos años después de adherir al Tratado de No Proliferación, reveló haber sido una potencia nuclear y haber desmantelado sus bombas nucleares debido al hecho de que entorpecían el desarrollo.

En conclusión, el derrumbe del muro nuclear ha disminuido la angustia del ciudadano mundial frente a la amenaza y ha reorientado los intereses de la mayoría de los Estados en cuanto a la obtención del arma nuclear como recurso de poder. Quedan planteados, sin embargo, los problemas derivados del control nacional, como ha sido detectado con evasiones desde Rusia; un mejoramiento del control internacional; mecanismos de seguridad regional; $y$, finalmente la cooperación entre los distintos Estados. Es en estos distintos puntos, que no son menores, donde quedan avances por hacer.

${ }^{32}$ Este aspecto todavia no ha sido suficientemente explorado. En esa dirección se ubica el trabajo realizado sobre los sistemas politicos de Canadá y México frente al proceso de integración. Vèase Lincoln Bizzozero, "Los partidos politicos de Canadá y México y el Tratado de Libre Comercio de América del Norte", Integración Latinoamericana, No204, Buenos Aires, octubre 1994. 
El incidente de las inspecciones nucleares con Corea del Norte, se inserta en los problemas que pueden plantearse en el futuro. El acuerdo firmado recientemente, permite disipar el engranage de una crisis cuyas aristas y resolución no hubieran sido claras. En efecto, en esa situación, con el respaldo tácito o expreso de China a Corea del Norte, ¿qué solución hubiera sido posible? De vuelta, nos encontramos con el callejón sin salida de los intereses nacionales en el sistema intemacional y de las consecuencias y costos a asumir en distintos escenarios conflictivos del plano "estratégico-militar".

Estas distintas rendijas y brechas que quedan en materia de control nuclear, ponen al descubierto las dificultades de gestionar la creciente interdependencia entre los Estados. De todas formas, las derivaciones del dernumbe del muro nuclear han sido importantes en los distintos planos analizados.

\section{Las aproximaciones.}

En este plano ubicamos el derrumbe de dos muros sólidamente establecidos: el vinculado con las relaciones Vaticano-Israel y el vinculado con la integración entre países de distinto nivel de desarrollo.

El acuerdo fundamental, firmado el 30 de diciembre de 1993, entre el sub-Secretario del Consejo para las relaciones de la Iglesia con los Estados, Monseñor Celli, y el vice-Ministro de Asuntos Extranjeros del Estado de Israel, Yossi Beilin, fue la culminación de un proceso que comenzó con la visita del Papa Paulo VI a Jerusalem y a Tierra Santa. ${ }^{33}$ Sin embargo, en los últimos años los acontecimientos se precipitaron con vistas a una normalización. La influencia de la Iglesia Católica en una parte de la población palestina, su reivindicación frente a la comunidad internacional para obtener una solución al problema creado con Jersusalem, el interés religioso en la zona y la ausencia del Vaticano en el inicio de las negociaciones multilaterales árabe-israelitas, explican el momento de la concreción del acuerdo.

En lo que concierne al sentido de la aproximación posterior al acuerdo, interesa específicamente el hecho de que está prevista la designación de comisiones que regularán aspectos prácticos de la vida religiosa. Esta aproximación entre dos concepciones religiosas permitirá en definitiva definir aspectos prácticos como los peregrinajes, el estatuto de

\footnotetext{
${ }^{33}$ Véase el artículo de Francesco Margiotta Broglio, "Israel-Vatican: un accord historique entre espoirs et craintes", Géopolitique, N²5, Paris, primavera de 1994.
} 
los organismos católicos, las propiedades eclesiásticas y asegura el pluralismo religioso con vistas a futuras negociaciones. Esta aproximación tuvo continuidad con el establecimiento de relaciones diplomáticas, el 15 de junio de 1994.

Finalmente, la firma y posterior ratificación del Acuerdo de Libre Comercio de América del Norte permitió la aproximación de Estados con distinto nivel de desarrollo, descartando la idea de su imposibilidad a partir de la brecha económica que se expresa en los niveles salariales y de protección social. El Tratado de Libre Comercio de América del Norte (ILCAN o NAFTA, en inglés), intenta otorgar una respuesta a la problemática de la competencia proveniente de los paises del Sur, en determinados ramos de actividad, cuyos productos tienen un alto contenido incorporado de mano de obra: it:

Este problema constituyó uno de los temas novedosos incorporados a la agenda de la Ronda Uruguay del GATT. La resolución del mismo es fundamental en términos del futuro sistema de comercio internacional, tomando en cuenta la relación Norte-Sur. El TLCAN da una respuesta limitada y negociada, cuya base es la aceptación de la competencia de productos mexicanos y un ajuste intemo buscando un mejoramiento de la competitividad en la economía de Estados Unidos.

En definitiva, el ILCAN constituye una negociación de mayor alcance eri que se inscriben dos temas fundamentales: el de la migración proveniente del Sur y el de la competencia comercial. La resolución "negociada" del principio de la competencia de productos mexicanos en el mercado de Estados Unidos, permite abrigar expectativas moderadamente optimistas sobre el desarrollo de México y por ende servir como alternativa aperturista para los países del Norte. 34

Es en esa dimensión que debe entenderse el debate que se planteó en los Estados Unidos. La oposición de los grupos sindícales y ecologistas se proyectó al menor costo de los productos provenientes del Sur, debido a la escasez de controles sobre el ambiente y a la insuficiente protección salarial. El rechazo al proyecto del TLCAN por parte de Estados Unidos, hubiera continuado con la lógica de la interdependencia económica, sin que México contara con salvaguardias en la exportación de sus productos al mercado de los Estados Unidos. En definitiva, aun cuando resta un trecho grande por recorrer, la ratificación del Tratado firmado en 1992, plantea para Estados Unidos un necesario ajuste interno

${ }^{34}$ La reciente incorporación de Mėxico a la Organización de Cooperación y Desarrollo Económico (OCDE), no cuestiona la problemática conceptual sệalada en términos estructurales. 


\section{Bizzozero / Viejos y nuevos muros en el sistema ...}

en los ramos menos calificados y un mejoramiento de la competitividad en sectores altamente calificados por su creciente productividad. ${ }^{35}$

\section{II.- Edificaciones y bloqueos.}

En el punto anterior nos referimos a distintos muros derrumbados con posterioridad a la Guerra Fría y a sus perspectivas renovadoras. Es así que fueron analizadas las reconstrucciones nacionales o en un marco estatal (Alemania, Africa del Sur, El Salvador, Mozambique, Camboya), la renovación de perspectivas en procesos regionales o mundiales de seguridad (Cercano Oriente, desame nuclear) y las aproximaciones dificiles que resultan cuando convergen dos perspectivas diferentes ya sean religiosas o económicas (acuerdo Vaticano-Israel y Tratado de Libre Comercio de América del Norte). En este apartado se analizarán en particular los distintos muros que han surgido con posterioridad a la Guerra Fría y a su propia dinámica de bloqueo: Estos nuevos muros son el "civilizatorio", el "nacional", el "Este-Oeste", el "Norte-Sur" y el que surge de la "amenaza interna".

Aun cuando los distintos muros indicados tienen su especificidad, pueden realizarse dos tipologías diferentes. La primera atiende a la relación de los muros con los paradigmas ordenadores en términos de sus tradiciones. En esa dirección ubicamos los muros que se relacionan con los paradigmas "tradicionales" y muros relacionados con paradigmas "postmodernos". Entre los primeros ubicamos los muros "nacional", "Este-Oeste" y "Norte-Sur", mientras entre los segundos ubicamos el "civilizatorio" y el de la "amenaza interna". La segunda tipología se remite a ubicar el origen del muro de acuerdo a la clásica división interno/internacional. En esta dimensión ubicamos los muros "nacional" y la "amenaza interna", que tienen su origen en un contexto estatal o nacional por una parte, y los muros "Este-Oeste" y "Norte-Sur" que se originan en el funcionamiento del sistema internacional. El muro "civilizatorio". participa por su especificidad de ambos.

${ }^{35} \mathrm{E} 1$ ajuste y sus costos originó ta oposición sindical al Tratado. México tenia frente a ello el arma migratoria. Más dificil desde el punto de vista de la negociación fue la oposición ecologista. 


\section{Muros y civilizaciones.}

El conflicto en la ex-Yugoeslavia, que comenzó cón reivindicaciones vinculadas con la identidad nacional y el derécho de los pueblos a su autodeterminación, constituyó el inicio de dos problemas diferentes. El primero se vincula con la persistencia de percepciones nacionales diferentes en Europa Occidental, que derivaron en la adopción de decisiones políticas divergentes con consecuencias regionales e impactos en la definición de políticas de seguridad europeas. El segundo problema se relaciona con la evolución del conflicto. El conflicto en la ex-Yugoeslavia comenzó con reivindicaciones de autodeterminación, se expresó posteriormente en conflictos nacionales entre repúblicas, continuó con una guerra "histórica" entre serbios y croatas, para luego degenerar en guerras de "depuración étnica". En esta última versión, la guerra se ubicó espacialmente en Bosnia-Herzegovina y tiene tres actores que combatieron entre sí: los serbios, los musulmanes y croatas. En el desarrollo de esta última versión del conflicto, hubo alianzas bilaterales para impedir la progresión del tercero. Ese fue el caso de las alianzas entre croatas y musulmanes, para contrarestar el avance serbio posibilitado por su mejor dispositivo bélico.

Esta guerra de tres actores en Bosnia-Herzegovina, se inserta en la línea de pensamiento de Samuel Huntignton. Para dicho analista, una de las líneas de fractura entre civilizaciones pasa por los Balcanes, lugar de confluencia de los imperios ruso, musulmán y de los Habsburgo. En términos históricos, para esta visión sobre el futuro de las relaciones intemacionales estos conflictos son milenarios: en el caso de la línea de separación con el mundo musulmán, remonta a su misma aparición.

Si nos atenemos a una versión histórica, aunque no se incluya la visión global recién señalada, el conflicto serbio-croata tiene orígenes antiguos, vinculados con la pertenencia civilizatoria. ${ }^{36}$ Es en esta dirección que la afirmación serbia por su autonomía se realizó en desmedro de poblaciones islámicas y turcas a partir de 1815. También la carga que tienen implícitos los mensajes de "purificación étnica" y "limpieza étnica" se inserta en anteriores escenarios de guerra. ${ }^{37}$

\footnotetext{
${ }^{36}$ Véase al respecto Julie Fournier, "Les antécédents du conflit yougoslave", Centre Québécois de Relations Internationales, "Le drame yougoslave", Universidad Laval, Quebec, 1093; y Michel Roux, "A propos de la "purification éthnique", en: "Bosnie-Herzégovine", Hérodote, N67, La Découverte, Paris, 1992.

${ }^{37}$ Todavia constituye un punto no resuelto politicamente el nưmero de serbios que fueron eliminados por los Oustachis croatas, en una política de exterminio masivo y dirigido. Véase al respecto, Predrag Simic, "Le conflit serbo-croate et l' éclatement de la Yougoslavie" y Christoper Cviic, "L' avenir incertain de la Croatie", Politique Etrangère, $N^{\circ} 1 / 94$, Paris.
} 
Esta continuidad latente del conflicto precipitó los acontecimientos que se conocen desde el año 1991. Sin embargo, a pesar de este conflicto latente y potencial, la salida pudo haber sido otra, como lo dejan entrever algunos analistas, frente al apresuramiento primero y la falta de convergencia y firmeza de Europa después. Por otra parte, la convivencia de distintas nacionalidades al interior de un Estado, aunque el mismo fuera comunista, permitía abrigar la expectativa de una solución negociada.

Los acontecimientos que se sucedieron con rapidez desde 1991, señalaron otro camino, a pesar de la intervención de Europa y de las Naciones Unidas. El muro "civilizatorio" se fue definiendo, sobre todo cuando la guerra se instaló en Bosnia-Herzegovina. El conjunto de mediaciones y planes de paz (plan Vance; plan Vance-Owen; plan Owen-Stoltenberg), que se fueron sucediendo, así como la intervención preventiva de las Naciones Unidas, no impidieron la edificación de este muro civilizatorio. En definitiva, el conflicto puso al descubierto las insuficiencias de las instituciones de prevención y cooperación europeas post-Guerra Fría. Las divergencias nacionales en materia de seguridad, la redefinición de los objetivos y funciones de las instituciones existentes, mostraron las ineficiencias europeas en la crisis de los Balcanes. Sin embargo, la necesidad de definiciones y las características desencadenantes de la guerra, tanto en un nivel espacial inmediato como en el nivel simbólico, por la imposibilidad de parar los exterminios étnicos, llevaron a un compromiso más firme de la Alianza Atlántica, en febrero de 1994, que tuvo su contrapeso en un posicionamiento ruso frente al conflicto. ${ }^{38}$ Este compromiso de la OTAN en términos de la prevención de nuevas masacres, con referencia a Sarajevo, no ha impedido la continuidad de la guerra y de delitos de lesa humanidad. Tampoco se ha avanzado en cuanto a la aceptación del último plan de paz propuesto, ya que las tres comunidades se consideran perjudicadas de diferente manera en ese reparto territorial. En efecto, los serbios han conquistado un $64 \%$ del territorio y la propuesta prevé el otorgamiento de $52 \%$; los musulmanes representan el $43,7 \%$ de la población y la propuesta le concede el $31,4 \%$ del territorio; $y$, finalmente, los croatas consideran que han sido perjudicados por la factibilidad de que se conforme la Gran Serbia.

El empantanamiento de las negociaciones y de la situación no hace otra cosa que adelantar las consecuencias que puede tener cualquier reparto y la pertinencia del muro civilizatorio para el futuro. Croacia se

\footnotetext{
${ }^{38}$ Para una visiön del conflicto en Bosnia-Herzegovina en los años 1993-1994, puede consultarse Hans Stark, "La catastrophe bosniaque", en: Thierry de Montbrial,Pierre Jacquet (dir.), op. cit.
} 
siente amenazada por la posibilidad de que se realice el advenimiento de la Gran Serbia; los musulmanes y los croatas se han visto abandonados por el mundo occidental; y los serbios confortados por el contrapeso eslavo de Rusia frente a Occidente, intentan definir y ampliar su patrimonio histórico.

El muro civilizatorio también tiene otras derivaciones post-Guerra Fría. La participación de las ex-repúblicas soviéticas del Asia Central en la Conferencia Islámica y la reciente realización de la primera cumbre turca, entre cinco de esos Estados ${ }^{39}$ y Turquía, han originado un alerta en Moscú, frente a esta reorganización del espacio turco en la zona Sur y Este de su territorio. La declaración de Estambul, resultado de esa primera cumbre, consta de 24 puntos, algunos de los cuales cuestionan los intereses ( $y$ el rol) de Rusia en la región del Asia Central. Entre esos puntos se encuentran: la demanda de un estatuto específico para el mar Caspio; la solicitud para que las Naciones Unidas y la Conferencia sobre Seguridad y Cooperación en Europa (CSCE) intervengan más activamente en el conflicto armenio-azeri; $y$, finalmente, el interés de encaminar sus recursos energéticos para el mercado mundial a través de la vía más corta. ${ }^{40}$ Por otra parte, es sintomático el hecho de que en la conformación de la Comunidad de Estados Independientes (CEI) el núcleo original estuviera conformado por los Estados eslavos y no haya participado ninguna de las repúblicas musulmanas ni del Cáucaso que pertenecieron a la Unión Soviética.

Finalmente, los actuales desempeños económicos de Asia han llevado a plantear la perspectiva de un equilibrio económico global entre la civilización occidental y la oriental para comienzos del siglo XXI. En esa línea de análisis, el equilibrio o la superación de Occidente por parte de Oriente puede tener una dimensión de "cruce pacífico", o bien hacer saltar las tensiones culturales y politicas. En definitiva, descartando la variable económica que puede atenuar o acelerar cualquier escenario, lo que importa es el hecho de la dimensión prospectiva de este equilibrio y su punto de partida. En la perspectiva del "final de la historia", el contexto civilizatorio queda subordinado a un destino que tiene contomos definidos, aun cuando puede haber conflictos relacionados con la definición de los espacios geoeconómicos. En una perspectiva civilizatoria, las

\footnotetext{
${ }^{39}$ Participaron cinco de las seis ex-Repủblicas soviéticas del Asia Central. Tadjikistän no participó en la cumbre porque tiene otras raices étnicas que pueden ubicarse entre los tadjiks de Irän.

${ }^{40}$ Estos distintos puntos plantean conflictos potenciales con Rusia y la orientación de la CEI. La intervención de las Naciones Unidas es rechazada por Armenia ( $y$ Rusia). La via corta de los recursos habla sin nombraria de Turquia.
} 
tensiones y conflictos se ubican desde sus orígenes, por lo que la posibilidad de amortiguarlos se relaciona con la confección de los canales adecuados. $^{41}$

En otros términos, aun cuando la interdependencia y la globalización económica se han afirmado como tendencias, la continuidad de las civilizaciones en su base cultural y religiosa ha facilitado la construcción del muro en distintas partes del mundo.

\section{Los muros de la real politik.}

La desintegración de la Unión Soviética hizo pensaren un primer instante en un vacío político con secuelas de conflictos nacionales y locales, entre las repúblicas y al interior de ellas. Esta visión estaba alimentada por el desarrollo que tuvieron las experiencias postcomunistas en los países de Europa Central y en Yugoeslavia. Esta visión no tuvo en cuenta los factores demográficos, territoriales y militares, que no dejában lugar a una evolución centifugadora. Por otra parte, la tradición histórica consituía un soporte de peso para esos tres factores.

Frente a esa visión, se contradecía otra que insistía en las capacidades de Rusia para seguir cumpliendo sus funciones en la estructura bilateral del sistema internacional. ${ }^{42}$ Entre la "explosión centrifugadora" y la "continuidad sistémica" hay una brecha grande, que incidirá necesariamente en las relaciones exteriores de los países de Europa Central y Oriental. En otros términos, la formulación de políticas para los países de Europa Central y Oriental se planteaba en distintos términos de acuerdo a los escenarios postdesmembramiento de la Unión Soviética: desagregación nacional, con derivaciones fuertemente conflictivas; formación de una asociación de Estados con vínculos cooperativos especificos; restablecimiento del poder de Rusia, en tanto eje centralizador y moderador del antiguo espacio soviético. Desde la perspectiva de Occidente, la política en relación a la ex-Unión Soviética privilegió tres objetivos con posterioridad a la caída de Gorbachov: apoyo "incondicional" a Yelstin en tanto representación de la legalidad del poder; apoyo a Rusia para controlar la transición post-Unión Soviética; y definición de

\footnotetext{
${ }^{41} \mathrm{El}$ interés por la seguridad regional ha aumentado en los últimos años. Ello se debe a preocupaciones legitimas relacionadas con la historia y los conflictos pendientes y al incremento del potencial militar de varios Estados. Uno de los actores centrales en esta nueva dinamica es China, cuyos movimientos son causa de preocupación. Véase el debate consagrado a China, "Faut-il avoir peur de la Chine?", Relations Internationales et Strategiques N¹5, Paris, 1994.

${ }^{42}$ Kenneth Waltz, "The Emerging Structure of International Politics", International Security, vol. 18, N2, Harvard, 1993.
} 
relaciones "occidentales" con algunos países de Europa Central (Polonia, Hungria). Esta definición de real politik por parte de Occidente se verificó en distintos momentos de la historia rusa presente. En el plano intermo, el conflicto de Yelstin con el Parlamento en octubre de 1993 llevó hasta un extremo el apoyo de Occidente. ${ }^{43}$

Más importante en términos de las consecuencias del desmembramiento de la ex-Unión Soviética en la posible edificación de nuevos muros, es la aquiescencia de Occidente (y de Estados Unidos en particular), frente a la definición de objetivos extermos por parte de Rusia. En ese sentido, luego de una corta etapa de "apertura incondicional" al Oeste por parte de Rusia durante el año 1992, las autoridades mostraron el nuevo consenso del gobierno en materia de política exterior: la estabilidad del espacio geopolítico que correspondía a la Unión Soviética es planteado como un asunto interno, que depende de la capacidad de Rusia. $^{44}$

En esencia, la doctrina rusa del "extranjero próximo" plantea los siguientes puntos: integración de la antigua Unión Soviética por parte de Rusia, como interés vital; seguridad de que el "extranjero próximo" no se asocie a países de Europa Central y Oriental para formar un doble cordón sanitario contra Rusia; y disponibilidad de los mecanismos necesarios a la Comunidad de Estados Independientes para cumplir esos objetivos a través del liderazgo de Rusia. ${ }^{45}$.

Las consecuencias de esta real politik en términos de un posible muro, tiene relación con la.configuración de esta nueva división EsteOeste, que ha comenzado a diseñarse. La mutua aceptación de las esferas de influencia en la reciente cumbre entre Clinton y Yelstin, es solamente el síntoma reciente de una situación que no permitió la adhesión de los Estados de Europa Central a la OTAN, sin la previa incorporación de Rusia. El veto de Rusia fue el preludio para su posterior adhesión a la

\footnotetext{
${ }^{43}$ Las posiciones de los países occidentales variaron desde el apoyo incondicional hasta un apoyo restringido por la falta de alternativas frente al posible caos que se produciria en caso de caer Yelstin. ${ }^{44} \mathrm{La}$ fecha exacta de esta definición depende de los análisis. James Sherr plantea cuatro etapas en la política exterior rusa a partir de 1990: la revolución (junio de 1990 hasta diciembre de 1991); la prioridad al Oeste (enero-octubre 1992); el retomo de Rusia al "extranjero próximo" (octubre 1992 hasta agosto 1993) y el retomo de Rusia al "extranjero lejano" (agosto de 1993 hasta el presente). Véase su artículo en la revista Géopolitique, $N^{\circ} 45$, París, 1994 . Otros análisis ubican el consenso entre Defensa y Relaciones Exteriores a fines de 1992 y su primera expresión oficial en febrero de 1993, en un discurso de Boris Yelstin en la Unión Civica. Cf. Marie Mendras, "Russie: le débat sur l'intérêt national", Problèmes Politiques et Sociaux, dic. de 1992; y "Le rẹtour de la Russie?", en: Thierry de Montbrial, Pierre Jacquet (dir.), op. cit.

${ }^{45} \mathrm{La}$ afirmación del rol de Rusia y de la CEI como institución regional parecen ser los resultados más destacables de la catorceava reunión realizada recientemente en Moscú.
} 
"Asociación para la Paz". en junio de 1994 y a un posterior acuerdo de cooperación con la Unión Europea. ${ }^{46}$

Aparte de las divergencias de los analistas sobre las interpretaciones de, fondo y las políticas a seguir, la necesidad sistémica de una reconstrucción sobre nuevos cimientos de un muro "Este-Oeste" tiene diversas facetas. En lo que se refiere a los países de Europa Central y Oriental, la sombra de no poder hacer determinados movimientos de política exterior fuera de los límites del muro. Para los países de Europa Occidental y sobre todo para Estados Unidos, la definición de condiciones y límites políticos y geográficos al renacer de'Rusia. En cuanto a Rusia y los países de la CEI, la edificación del nuevo muro y la continuidad del deterioro de la vida, sin canales apropiados para el desarrollo, pueden llevar a fortalecer los cimientos del muro, con consecuencias no previsibles en términos inmediatos para el resto de Europa. ${ }^{47}$

Entro los últimos años de la Guerra Fría y los primeros de la post-guerra, las potencias tuvieron que redefinir sus prioridades y su percepción global sobre los desafios inmediatos y las amenazas existentes. La redefinición de prioridades planteó el tema de la reconstrucción de Europa Central y Oriental en las transiciones políticas y económicas que tenían por delante. Esta prioridad que se configuró sobre todo para Alemania, los países de Europa Occidental y Estados Unidos, definió una fuente de conflicto con los países del Tercer Mundo debido a dos aspectos relacionados: la capacidad y vitalidad de la respuesta en la ayuda económica y la posibilidad de que esa situación originara un nuevo desplazamiento.

En otros términos, las nuevas definiciones de política exterior de las potencias, la inclusión de prioridades diferentes, las características y contenidos de los canales de cooperación económica, plantearon desde los inicios de los noventa el tema de las relaciones Norte-Sur. En definitiva, no podemos hablar en sentido estricto de un nuevo muro, sino más bien de las fronteras y modalidades del mismo, frente a las modificaciones del sistema internacional.

La idea básica de que el muro Norte-Sur se basa en un problema estructural, cuya brecha se ha ido agrandando y que representará el principal problema (y muro) para el sistema internacional de los próxi-

\footnotetext{
46 "La Russie adhère ai PFP", Revue de l'OTAN, N4, agosto de 1994.

${ }^{47}$ La historia de la transición económica de los paises socialistas se ha acompañado en todos los casos de una recesión y de una disminución del PBI del $10 \%$ al $80 \%$ en relación al año 1989 . Kolodko, G., "From Recession to Growth in Post-Communist Economies: Expectations versus Reality", Communist and Post-Communist Studies, vol. 6, N22, enero 1993.
} 
mos años, ha sido planteada por algunos autores. ${ }^{48}$ Sin embargo, aparte del hecho de que el denominado Tercer Mundo ya dejó de tener un contenido operativo en el sistema internacional ${ }^{49}$, los resultados políticos y económicos de algunos países de Asia y América Latina en los últimos años, aunado a su activismo en el sistema internacional, cuestionan desde la raíz la continuidad del muro "Norte-Sur", tal cual estaba concebido.

El tema planteado es entonces el de la nueva frontera "Norte-Sur", es decir el límite en que las consecuencias del nuevo posicionamiento del sistema internacional, se hacen palpables. En ese sentido, es que puede señalarse que las nuevas fronteras se ubican en el Africa profunda, tanto por su fragilidad estructural como por su endeblez institucional. Esta frontera deja afuera al Africa mediterránea, espacio prioritario para Europa, así como Africa del Sur, que como ya se indicara constituye la posible altemativa para la récúperación económica de la región.

Es entonces en el centro de Africa que las consecuencias de esta nueva situación se han manifestado de manera impactante. Los muertos en las guerras civiles, étnicas o tribales se cuentan por millones; las epidemias, pestes y hambrunas diezman a poblaciones enteras; los refugiados políticos o económicos son multitudes; y las acciones de depuración o desplazamiento colectivo constituyen un argumento común. ${ }^{50}$ En definitiva, las consecuencias post-Guerra Fría en Africa, reafirman la denominada "desconexión", que se verifica de distintas formas y en diversos planos. ${ }^{51}$

A este primer sentido del muro referido a las consecuencias estructurales, hay que agregarle el que derivó de la reformulación de la amenaza externa. En ese sentido, la guerra del Golfo Pérsico confirmó la evolución de un miedo indefinido al Sur en el imaginario colectivo de los países de Occidente. ${ }^{52}$ Esta percepción indefinida de peligro del Sur; se alimenta con temas que están presentes en la agenda internacional y continental, como el relacionado con las migraciones, el tráfico de droga y el de armas. Esta derivación del muro "Norte-Sur" no se ha confirmado en estos últimos años, por el hecho de que se ha producido una canalización

\footnotetext{
${ }^{48}$ Franz Nuscheler, "El conflicto Norte-Sur..." op. cit.

${ }^{49}$ Indudablemente constituyó un marco conceptual, que permitió determinados avances. Sin embargo, desde mediados de los setenta su lógica abarcadora terminó siendo un freno en los intentos de definir los problemas y soluciones especificos.

${ }^{50}$ Philippe Leymarie, "L' Afrique appauvrie dans la spirale des conflits", Le Monde Diplomatique, sept. 1994. Para un análisis particular de los conflictos en Rwanda y Burundi, véase en el mismo número, Colette. Braeckman, "Le feu court sur les Grands Lacs".

${ }^{51}$ Daniel Bach (dir), "Afrique: la déconnexion par dëfaut", Etudes Internationales, vol. XXII, N2, junio 1991, Universidad Laval, Québec, Canadá.

${ }^{52}$ Cultures \& Conflits, $N^{2} 2$, "Menace du Sud: images et réalités", L' Harmattan, Paris, primavera 1991.
} 
de estos temas en las respectivas esferas regionales, por una parte, y por un cambio sensible en la política de Estados Unidos, por la otra. La aprobación del Tratado de Libre Comercio de América del Norte y una reorientación del presupuesto dedicado a combatir la droga en el lugar de consumo, han sido los indicios de esta reorientación.

\section{Muros centrifugadores y restauradores.}

Entre los muros provenientes del sistema intemo que tienen efectos centrifugadores, encontramos el "nacional" $y$ algunas modalidades referidas a la "amenaza interna". A su vez, este último muro cumple en ocasiones funciones.restauradoras. El primero de los muros está asociado a todos los movimientos, solicitudes y desafios que produce cualquier cambio mayor en el sistema internacional. En ese sentido, no hay diferencias fundamentales entre las actuales solicitudes de autodeterminación de los pueblos, de reivindicaiones nacionales y de derechos históricos, como la ocurrida al final de la Primera Guerra Mundial.

El muro de la amenaza interna tiene en cambio cimientos originales, que se produjeron a raiz de las consecuencias del dernumbe de los regímenes socialistas. La denominada "amenaza interna" tiene dos derivaciones: la referida al peligro de utilización del aparato estatal y la señalada con la reorientación presupuestal de defensa hacia el marco interno. El peligro de utilizar el aparato estatal por parte de grupos organizados y mafias ocasiona insuficiencias crecientes del Estado en cumplir sus funciones mínimas de seguridad y de legitimidad Jel sistema, con consecuencias evidentes en la sociedad y el ambiente externo. Por otra parte, esta modalidad también puede llevar a persecución de ciudadanos, grupos o etnias, creando muros internos con posibles efectos centrifugadores. Los ejemplos de ambas modalidades de utilización del aparato estatal son legión. Los múltiples fenómenos de corrupción en diferentes países, las conexiones entre la mafia y el sistema político y las derivaciones en canales transnacionales de ventas de armas y narcotráfico, están planteando drásticamente las insuficiencias del Estado. ${ }^{53}$ Uno de los casos particularmente grave es Rusia, donde se estima que los diferentes grupos mafiosos dominan el $40 \%$ de la economía del país. 54

\footnotetext{
${ }^{53}$ Didier Bigo, Michel Dobry, "L'interpénétration des jeux mafieux et politiques", Cultures \& Conflits, NO3, "Mafia, drogue et politique", oton̄o 1991; Daniel Hermant, "Les rëseaux internationaux de violence", Cultures \& Conflits, $\mathrm{N}^{\circ} 4$, invierno 1991-1992.

${ }^{54}$ Nicole Gnesotto, "Deux fois deux mondes", en: Thierry de Montbrial, Pierre Jacquet (dir.), op. cit, donde se refiere a un informe de la Central de Inteligencia de Estados Unidos.
} 
La otra modalidad del muro de la amenaza intema va exactamente en la otra dirección de esta posibilidad y tiene relación especialmente con una reorientación del presupuesto de defensa para combatir la criminalidad al interior de los países. Esta incipiente tendencia se visualiza con fuerza en Estados Unidos, con la reciente aprobación de la ley de control de la venta de armas y con el proyecto de ley sobre la criminalidad, que llevaría a incrementar los recursos de la policía en cien mil hombres, con mejoras en el presupuesto y la realización de más prisiones. ${ }^{55}$

Esta reorientación para depurar la sociedad con un mayor control y poder del Estado puede derivar en muros internos, si no se toman otros recaudos y no tendría por qué derivar hacia el sistema internacional. Sin embargo, la interdependencia en términos de flujos de corrientes de ideas y modalidades institucionales es creciente, por lo que no es de extrañar una sucesión de peripecias restauradoras que puedan tener efectos discriminatorios para distintos sectores de la población mundial. ${ }^{56}$ Otro ejemplo con sentido restaurador, aun cuando con un perfil diferente y cuyas consecuencias dejarán secuelas de otro orden, es el actual proyecto de ley a aprobación en el Parlamento ruso, que impide todo ingreso al país de portadores de SIDA o de quienes no se presten a la realización del examen correspondiente. Las restauraciones nacionales de estas características, se ubican claramente en los cimientos de un muro discriminador hacia diferentes grupos de personas, etnias o naciones.

\section{III.-Perspectivas.}

Después de enumerar los muros derrumbados y los novedosos bloqueos que han surgido en el sistema internacional, resulta de interés reflejar la situación de América Latina en ese contexto. Una de las primeras consideraciones, es que América Latina en esta enumeración se ha visto beneficiada de esta inmediata recomposición post-Guerra Fría. En pri-

\footnotetext{
${ }^{55}$ Una de las bases de la nueva ley sobre la criminalidad en los Estados Unidos es "three strikes and you're out", por la cual una persona que cometió tres infracciones es condenado a prisión perpetua. Esta medida contenida en la ley ha sido denunciada y cuestionada por las consecuencias raciales que apareja. Véase Roseylyne Pirson, "Surenchère répressive et surveillance des pauvres", Le Monde Diplomatique, octubre 1994.

${ }^{56} \mathrm{Las}$ colonias de inmigrantes constituyen uno de los sectores donde canalizar las medidas restauradoras. En ese sentido, la aprobación del plebiscito en California, por el cual la ley 187 (Save Our State), excluye los inmigrantes ilegales (se calcula que son un millón y medio), de todos los programas sociales, médicos y educativos del Estado de California. Ello provocó juicios duros de Carlos Salinas de Gortari, Presidente de México en ese momento, sobre la intolerancia y la exclusión de la educación de miles de niños.
} 
mer lugar, algunos muros políticos derivados del enfrentamiento EsteOeste han tenido solución y una región como América Central puede enfrentar los nuevos desafios con bases más estables derivadas de la pacificación. Indudablemente, si tomamos el conjunto del Caribe debamos hacer la excepción de Cuba, como también lo fuera a fines del siglo pasado. En segundo lugar, en el debate por insertar a la región en el sistema económico internacional y por lograr bases multilaterales más firmes en el comercio internacional, Estados Unidos aceptó el principio de incorporar un país de distinto nivel de desarrollo al Tratado de Libre Comercio de América del Norte. En tercer orden, el asentamiento de democracias, las reformas económicas encaradas y los resultados macroeconómicos de los últimos años, permiten encarar una nueva era de panamericanismo. Es en esa dirección que debe entenderse la Cumbre de las Américas, aun cuando, de vuelta como en el pasado, los objetivos hegemónicos de Estados Unidos puedan llevar a una reacción de los Estados latinoamericanos, o al menos de los que componen la frontera Sur de América del Norte, lo que excluiría a México. Finalmente, en los nuevos muros, ninguno de los enumerados conciemen directamente a América Latina en el presente, a pesar de su sombra futura. Ello es así sobre todo en caso de una descomposición del escenario internacional, con posibles repercusiones en diversos planos. Aun cuando ello no es de descartar, los desafios planteados en el trabajo parecen encaminarse hacia otras variantes.

En definitiva, aun cuando la lógica división intemo/internacional se haya estrechado, no es actualmente en el sistema internacional que debamos ver muros que nos provoquen el pesimismo sobre el futuro de América Latina. Ello es así en relación a los puntos centrales de este trabajo, por lo que los efectos negativos de una culminación inadecuada de la Ronda Uruguay del GATT en el sector agrícola y la continuidad de la deuda externa no son capaces de generar de por sí los cimientos de nuevos muros. En cambio, las lógicas internas, tanto en términos de evoluciones políticas como de insuficiencias estatales y articulaciones societales, plantean dudas en cuanto a las perspectivas de un optimismo sobre la evolución de América Latina en el sistema intemacional y sobre su posibilidad de participar en los fundamentos de base que tendrá en el siglo venidero. En otras palabras, son algunos de los muros internos de América Latina, en cuanto a los poderes paralelos, a las brechas societales, a los porcentajes de su pobreza absoluta y, sobre todo, a la desconfianza en sus instituciones y en la capacidad de renovación de los contenidos y formas de la política, que pueden llevar a que se erijan algunos muros segregadores del continente en el sistema internacional. 
En cuanto a las conclusiones generales, las notas de optimismo que trajeron las reconstrucciones de Alemania, Africa del Sur y varios países en la lógica de guerra ideológica-política y el proceso en el Cercano Oriente, han sido contrarrestadas por la guerra en los Balcanes y el drama del Africa profunda. En lo inmediato, de todas formas las razones hacia el optimismo inclinan la balanza, aun cuando esta situación de "indefinición estratégica" no pueda durar muchos años. En otras palabras, la reestructuración de los espacios políticos y geoeconómicos darán cuenta de un nuevo equilibrio estratégico. Mientras tanto, Estados Unidos buscará continuar gestionando su primacía, aun cuando ello implique nuevos puntos de fractura que se expresarán en el dominio económico, tecnológico y político.

En lo que concieme a los nuevos muros, su consolidación se hará efectiva en los próximos años y dará cuenta del optimismo post-Guerra Fría. En esta perspectiva, la intolerancia en relación a la primera visión del "Otro" ha ganado terreno en diversas partes del mundo, a pesar de la globalización económica.

En definitiva, lo que hay que tener en cuenta es la conjunción de una globalización económica, de un incremento del regionalismo y de una continuidad de los espacios nacionales. Es por ello que adquieren fundamental importancia las modalidades y contenidos de estos nuevos muros y su posible consolidación. $\mathrm{Y}$ también por ello resultan más fundamentales que nunca estos años de final de siglo para América Latina. 